\title{
Urgences
}

\section{Madeleine Gagnon : conjuguer les temps d'un texte \\ Madeleine Gagnon, Les fleurs du catalpa, Montréal, VLB éditeur, 1986, 129 p.}

\section{Paul Chanel Malenfant}

Numéro 15, octobre 1986

Épigraphiques

URI : https://id.erudit.org/iderudit/025361ar

DOI : https://doi.org/10.7202/025361ar

Aller au sommaire du numéro

Éditeur(s)

Urgences

ISSN

0226-9554 (imprimé)

1927-3924 (numérique)

Découvrir la revue

Citer ce compte rendu

Malenfant, P. C. (1986). Compte rendu de [Madeleine Gagnon : conjuguer les temps d'un texte / Madeleine Gagnon, Les fleurs du catalpa, Montréal, VLB éditeur, 1986, 129 p.] Urgences, (15), 107-111. https://doi.org/10.7202/025361ar d'utilisation que vous pouvez consulter en ligne.

https://apropos.erudit.org/fr/usagers/politique-dutilisation/ 
alouettes, tombe sur la page comme dans l'horizon plat du scintillement évidé. L'étoile trompe le tain où se mire la lectrice, déborde le cadre, met "knock-out", n'offre rien à lire en sus, trahit la vérité.

De nature métalinguistique, le texte d'André Gervais se sert de la langue comme jeu d'exploration, de vertige et de jouissance pure. Comme les personnages de Carroll, il affiche le plus grand mépris pour les règles de la communication phatique. Le monde s'y articule en fraude en sa syntaxe et en son lexique. L'écriture est un corp(arcour)s (p. 28), elle établit un brouillage ne permettant plus de distinguer ce qui relève de la parole, des lettres ou du corps. Les mots y ont un sexe. Ils habitent leurs actes, leurs états d'encre (p. 20).

\section{encré d'aines outrefoutre, incrusté de coquilles outremer, de mots apocryphes à toison noire, déchairure iggervée, d'humus vire-gueule, dans les grammaticallosités l'astérité (p. 28).}

Le langage ne vit pas sans ces bonds hors de soi dont l'élan fait l'espace. Le langage ne se contente pas d'être pour l'artiste, il doit créer pour faire exister ce vide où il nous jette. Dès le principe, en création, il y a délai et distance, de quoi permettre au langage d'être ailleurs qu'en soi, autre que soi. Pourtant, en une certaine poésie modeme, le langage ne s'exile pas de soi, choisissant de s'entendre du fond de son absence, pour lentement revenir sous forme nouvelle (aussi loin que possible du chant innocent et mélodieux de Mendelssohn ou de César Franck). Une certaine poésie moderne s' oblige au saut périlleux verbal où rien n'est sacré et surtout pas cette "drogue génératrice d'habitude" qu'est l'art. Knockoutant les icônes esthétiques, elle permet ainsi à toutes les portes de rester ouvertes et fermées. Humour et scepticisme y offrent des possibilités de rester libres. Un certain CHAR Y VA:RIS! c'était la façon même de Duchamp.

Athée, la langue d'André Gervais nie, pour percevoir sa propre odeur, remonter de ses pius obscurs confins, de l'invincible usure de soi, de la perdition de la vie où écrire, c'est faire mourir, pas tuer, mais faire mourir: où écrire, court-circuitant deux incipits (p. 7 et 17), it's me, mots et mort.

Jocelyne Felx

\section{Madeleine Gagnon: Les fleurs du catalpa, Mon- tréal, VLB éditeur, 1986, 129 p.}

\section{Madeleine Gagnon: Conjuguer les temps d'un texte}

On connaît déjā la fonction de métaphore emblématique des titres en regard des textes qu'ils informent et dont ils sont informés, leur valeur conjointe de générateur et de condensé de la signification à l'égard des motifs et des dispositifs structuraux que l'oeuvre met en place. Madeleine Gagnon, dans son dernier recueil, Les fleurs du catalpa, utilise à fond la rentabilité signifiante de l'indicatif titulaire; en effet seize titres ponctuent le livre et en articulent l'itinéraire: 1 'L'étrange figure du lent destin des 
choses". 2. "En passant". 3. "Vision". 4. "Les fleurs du catalpa". 5. "Gestalt". 6. "Décembre". 7. "Janvier-février". 8. "Un chat blanc sur le tapis de neige". 9. "Un jour, la ville". 10. "Étrange filiation". 11. "Le corps ne pense plus". 12. "Orientation". 13. "Parler à partir du seul silence". 14. "L'année suivante exactement". 15. "Pour avoir déjà conçu la vie". 16. "Par ce long détour se pense la fin". Chacune de ces parties est datée (la première de décembre 1982, la dernière de févier 1986), datation qui désigne la ponctualité de l'écriture et qui, en même temps, inscrit la durée matérielle de la rédaction du livre. En outre, intrinsequement, chaque section du recueil tient à la fois de la "suite", par le nombre de poemes qu'elle regroupe, et du "moment" poétique, par la correspondance voire la connivence thématique et formelle qui caractérise les textes de telle série. Ainsi, structuralement, dans son déroulement linéaire, le poème est sériel, donc continu, alors que le découpage du livre qu'instaurent les titres travaille la fragmentation ou plutôt une segmentation insulaire de la partie à l'égard du tout.

Ces observations sont déjă rêvélatrices de l'adéquation entre le propos temporel du recueil et son organisation factuelle, qui opere simultanément dans la durée et la rupture textuelle; par ailleurs, on aura sans doute remarqué comment, du point de vue syntaxique, la plupart des titres relèvent de la phrase nominale - icône grammaticale de "l'instant", de la fraction saisie et nommée d'un capital temporel incalculable - (on sait, de plus, l'efficace poéticité de la nomination) et de la proposition infinitive ou participiale qui tiennent le sens - et le "temps" continu - en suspens; du point de vue sémantique ces titres montrent tour à tour des états présents ou existentiels, des motifs spatiaux ou climatiques et ils rejoignent ainsi la description condensée du procès d'écriture de l'auteur qui apparait à l'endos du livre:

Poèmes du temps présent. Le temps de l'heure et le temps qu'il fait. Livre d'amour écrit au fil des jours, au gré de la température, dans une terre d'exil, de glace et de tendresse: L'écrit est fidele à ce qui se donne dans la présence. Remontée dans le temps tout aussi nécessaire: avec la profondeur du vaste champ de la lettre. (je souligne)

Notons encore que deux des titres s'énoncent à l'indicatif présent, "Le corps ne pense plus" (11) et "Par ce long détour se pense la fin" (16), qu'ils affirment, l'un la provisoire inertie du cogito, l'autre l'achèvement et la perte perpétuelle de la pensée poétique, enfin que tous deux, par ce travail simultané de la continuité et de la dissémination contrapuntique du "même", font écho, dans le temps et dans le "coeur de la lettre", à un précédent livre de l'auteur, Pensées du poème (VLB éditeur, 1983).

Enfin, le titre fondateur du recuell, dont la sélection et l'importance sont attestées par la présence de la partie éponyme ("Les fleurs du catalpa" est la quatrième partie d'un livre qui en compte seize; elle est donc située stratégiquement au premier quart du parcours textuel), pose aussi, sémantiquement, la problématique temporelle qui fonde ce livre de Madeleine Gagnon. En effet "les fleurs du catalpa", outre qu'elles supportent, par le recours à l'arbre qui les détermine, les valeurs symboliques traditionnelles de la durée et de l'éphémère, constituent aussi, dans le schéma arborescent, les parties quintessenciées d'un ensemble végétal; elles "sont", à la fois dans l'autonomie de leur singularité comme dans le foisonnement de leur appartenance à un tout, bref elles rappellent simultanément l'unité de la constellation de l'arbre et le privilège unique d'une fraction florale close. La fleur se donnerait donc comme l'instant en regard de la durée de l'arbre en même temps que, spatialement, elle accomplit sa plus extrême présence à la faveur du "laps" ou de l" "entre" qui l'isole de ses semblables.

Nous sommes très près ici, par représentation figurative et à la lumière de tous 
ces autres indices textuels qui stipulent une continuité brisée, puis reprise, de la bachelardienne "intuition de l'instant", induite à partir de ce constat: "[...] le temps est une réalité resserrée sur l'instant et suspendue entre deux néants"'. Ainsi, dès le premier texte du livre où s'engage la perception de la durée dans le temps de l'écriture, dans celui de la conscience d'un être et d'une pensée qui s'absentent, Madeleine Gagnon écrit:

Le temps coule parfois, ne se compte pas, comme si j'écrivais un peu: je ne pense pas et je suis. [...] Rien ne se détache du tout. Chaque parcelle pourtant, sans rien liurer, semble douée d'éternité. [...] Tu dis alors: je suis celle qui est dans la parole absolument, ceci est mon corps, ceci est mon sang, dans les siècles des siècles. (p. 9).

Dilemme oxymorique quant à la saisie du tout et du rien, du fragment et de l'ensemble, de l'instant et de l'éternité. Il s'agit alors pour le poète de cerner le laps, de capter tel état de latence ou de lenteur, de sonder ces lieux de l'entre et de l'interstice, de dire, dans le corps de l'écriture, la pause ("Quand le poème éclate, ou se fragmente, ne pas prendre le vide d'assaut avec les armes du discours. Respirer dans les pauses. Parler à partir du seul silence." (p. 96)) et le suspense du sens: "Soudain un sens suspendu, un suspense, dans le surprenant minuit éclate la noirceur du monument." (p. 12).

Le "réel absolu" advient dans la plus immédiate présence d'un langage en suspension et le poème se constitue lui-même en un espace-temps ("une page veille/ attentive/au moindre mouvement/du temps/dans la ligne/continue" (p. 81)) et un espace-texte tant il est vrai que "[...] l'écriture est un calque du contour des objets, imaginés, du temps gagné sur l'heure pour en savoir bientôt la suite. Le présent nommé se trouve déjà passé: une extension fictive de ce qui a été." (p. 11).

Si la réflexion temporelle se situe au centre du recueil, qu'elle l'investit de la tension même d'une impossible saisie, l'écriture se refuse pourtant à la seule béance du constat abstrait. Au contraire, l'auteur excelle à rendre "l'air du temps", à prendre "l'instant aigu" comme un instantané photographique: "Un seul moment, celui-ci/le souvenir d'une glace givrée dans l'arbre/d'une lionne ailée volant d'un pic à l'autre/tout regard, tout animal/et ce désir génial du verbe/le parfum de verveine/etl'enchantement/ du velours, de l'eau, du piano" (p. 42). Ici, la sensation fait sens et "dire je suis" (p. 43) conjugue "je sens" et "je pense". Cela donne encore ces textes miniatures, clichés vifs où se concentre le sens, où se conjoignent le choc et l'éclat de voix. Minute de vérité: "Une fraction de seconde/dans la toundra du coeur/tu es partie/ne laissant rien au hasard/aucun enfant derrière toi" (p. 17). Le fait divers, le geste posé, l'anecdote du quotidien sont investis, avec l'ironique ingénuité d'un retour à l'enfance - ce temps où le temps même se nie -, d'un relief inédit: "Puis de toutes pièces et toujours de rien/de presque rien:/d'un mur de briques, d'une tenêtre givrée/d'un fil électrique, d'un pneu dégonflé/d'une minoune rouillée, d'une neige infinie/ils créeront un lieu/féérique, fabuleux/comme celui des Enfants terribles" (p. 27).

Devant la vacuité ou l'indigence étale des choses, le poème se donne comme autre chose, il se définit lui-même comme lieu de langage inusité, comme événement ex nihilo. Tel Dieu, tel l'enfant (à l'instar de cet enfant-dieu déjà exalté par Chamberland) le poète invente "toujours tout à partir de rien" (p. 34).

Alors l'état privilégié de l'écriture réside dans l'éclat, l'éclatement. l'éclaboussure: "Dire je, à toi, dans les éclaboussures de l'être" (p. 35). Puisque tout rassemblement est parcellaire, puisque la ligne du temps est une ligne brisée par un laps de néant 
dans la succession des instants, l'oeuvre même consent à l'écoulement, a la dissolution et au fracas: "Dans la matière opaque s'est dissous le poème [...] Alors, tout recommencer comme à partir de rien (on dirait): à partir du fracassement de la plerre [...], à partir de son émiettement et ses fleurs d'étincelles, son éclatement" (p. 57). Devant le fractionnement du réel, le texte parle l'effraction, la contamination des genres, le trompe-l'oell: "Toute écriture une effraction, tu as semé la règle du roman aux vents chauds du poème. [...] Toute écriture est apocryphe". (p. 13). Cet exercice d'une parole de la catastrophe, supporté par diverses techniques de dissémination, de morcellement textuels, et si fréquent dans la poésie québécoise contemporaine (de Roland Giguère à Pierre Nepveu, de Fernand Ouellette à Claude Beausoleil...), semble bien découler d'une conscience exacerbée de la virtuelle pulvérisation du monde. Et ainsi, chez Madeleine Gagnon: "L'ombellifere des mots ne changera pas grand chose à la puissance des astres dans leur fractionnement nucléaire. Éblouissement." (p. 57). Par ailleurs, chaque poète sait trouver, dans la dynamique même de son oeuvre, - "La seule folie, l'absence d'oeuvre" (p. 35) - les données conciliatrices qui viennent pallier les figures du désastre, combler les manques, les blancs:

La poésie se crée aussi dans les blancs, dans les fragments d'absence. Le tableau est un fragment. Mes écritures comprennent des fragmentstableaux. La pause fait partie de la ligne mélodique. L'harmonie donne un sens à ces multiples lignes, ces infinis fragments. L'écriture d'une femme, amante, mère et ménagère est forcément fragmentaire. C'est une question de temps, de rythme, de rapport au temps de la gestation. (p. 97).

On aura compris comment, chez Madeleine Gagnon, le fragment s'admet comme pulsion méme de l'oeuvre et de la connaissance, qu'il est "autre" dans la simultanéité de sa dissemblance et de sa ressemblance avec "je", ainsi, qu'il trouve son sens dans une ouverture à l'altérité dont la maternité constitue l'expérience la plus achevée: "La mère est cet autre" (p. 69) et [...] je rêve à mon enfant qui m'a ouvert le corps vers l'autre absolument. Comme une nappe infinie d'enfance éternelle." (p. 86). Il s'agit bien, puisque la langue, là où ça parle, est maternelle, de "Fouiller l'écrit/comme on cherche la mère". (p. 73).

Une femme ici (s')écrit; nulle polémique pourtant n'est engagée à l'égard de ces dites prérogatives paternelles du pouvoir, vis-à-vis de ces séculaires complexes d'auteur et d'autorité: "Mon père fut aussi le premier à me parler du nom, mais aussi de la signature et le Nom-du-Père pour moi ne fut jamais réductible à l'Ordre-de-la-loi ou au Pouvoir seulement" (p. 97). L'engagement de l'écriture, s'il en est, se désigne en une toute autre voix. Le roman familial s'ouvre à l'histoire et l'altérité se porte jusqu'à la collectivité, jusqu'à une franche empathie à l'égard de ce "Juif de Pologne" (p. 31) hospitalier dans sa boutique; car "Par la question juive sans réponse, je m'oriente dans le livre, le mien. Notre Orient le plus proche, fils et mère, est contenu dans la question juive sans réponse. Nous tenons nos doutes de cette proximité à cet exil." (p. 87)

Oeuvre altruiste, humaniste, liure d'orientation dans le temps et l'espace: ces accents se conjoignent et se fondent dans le texte rassembleur de Madeleine Gagnon par l'aveu constant d'une passion de vivre et d'écrire. Au "vécrire" de Jacques Godbout, au "Écrire est un grand amour" de Hubert Aquin fait écho cet "amour incommensurable d'écrire/ce temps présent" (p. 125). L'écriture comme sujet d'elle-même, cela s'écrit-il encore; et qu'advient-il de sens à interroger le sens? Le poète, au fil de son parcours, sans cesse se pose la question: "Que signifie ton livre? Le poète répondrait: je n'en sais rien, seul le livre le sait." (p. 11) Au lecteur, donc, de réécrire l'oeuvre, en y 
prenant "connaissance", en la lisant.

Si à la "pensée du poème" a succédé la "pensée de l'oeuvre" c'est bien que le poème se donne comme une oeuvre unique, l'oeuvre, comme un poème multiple. Madeleine Gagnon franchit ici la distance qui a la fois isole et rassemble la page et le livre, elle accorde à la solitude multiple, le privilège d'être unique multitude: "Car écrire est un déploiement de solitude peuplée d'autre et d'au-delà" (p. 112). Enfin, en témoigne la linéarité rompue du texte qu clôt le recueil, elle instaure la contiguité du mot à mot et du phrasé, elle accorde, dans ce suspense temporel propre à toute écriture, l'instant fui et la durée en-allée:

\begin{tabular}{|c|c|c|}
\hline Amour & $\begin{array}{l}\text { Et mon chemin est antérieur } \\
\text { L'écriture en toutes formes }\end{array}$ & L'écriture \\
\hline $\begin{array}{l}\text { en } \\
\text { de l'autre } \\
\text { tout-autre }\end{array}$ & tous temps & passage \\
\hline
\end{tabular}

février 1986 (p. 129)

1. Gaston Bachelard: L 'intuitton de l'instant, coll. "Bibliothèque Médiations". Paris. Éditions Denoël Gonthier. 1979. p. 13. 